\title{
Intraday Variability and Microarcsecond Structure in Blazar Cores
}

\author{
David L. Jauncey ${ }^{1}$, Lucyna Kedziora-Chudczer ${ }^{1,2}$, J.E.J. Lovell ${ }^{1}$, \\ Jean-Pierre Macquart ${ }^{3}$, George D. Nicolson ${ }^{4}$, Rick A. Perley ${ }^{5}$, John E. \\ Reynolds $^{1}$, A.K. Tzioumis ${ }^{1}$, Mark A. Wieringa ${ }^{1}$, Hayley E. Bignall ${ }^{6}$ \\ ${ }^{1}$ Australia Telescope National Facility, CSIRO, Epping, NSW, Australia \\ ${ }^{2}$ Anglo-Australian Observatory, Epping, NSW, Australia \\ ${ }^{3}$ RCfTA, School of Physics, University of Sydney, NSW, Australia \\ ${ }^{4}$ Hartebeesthoek Radio Astronomy Observatory, Krugersdorp, South \\ Africa \\ ${ }^{5}$ National Radio Astronomy Observatory, Socorro, NM, USA \\ ${ }^{6}$ Department of Physics and Mathematical Physics, University of \\ Adelaide, SA, Australia
}

\begin{abstract}
The accumulation of evidence now strongly favours interstellar scintillation (ISS) as the principal mechanism causing intra-day variability (IDV) at $\mathrm{cm}$ wavelengths. While ISS reduces the implied brightness temperatures, they remain uncomfortably high. The distance to the scattering screen is an important parameter in determining the actual brightness temperature encountered. The high brightness temperatures, the presence of strong and variable circular polarization and the observed lifetimes of a decade or more for several IDV sources, pose significant problems for synchrotron theory.
\end{abstract}

"The fault, dear Brutus, is not in our stars, but in ourselves, that we are underlings."

William Shakespeare, Julius Caesar

\section{Introduction}

Observations of the variability of extragalactic radio sources at radio wavelengths have demonstrated the existence of a variety of scattering screens between the observer and the sources. Early observations of variability at metre wavelengths were shown to be the result of scattering in the ionosphere and were not intrinsic to the source. As a result, Ginsburg (1956) suggested that the irregularities in electron density not only in the ionosphere but also in the interplanetary medium and the interstellar medium (ISM) should also give rise to detectable scattering and hence apparent variability in extragalactic radio sources. Interplanetary scintillation (IPS) of compact sources at decimetre wavelengths was found soon after and proved to be a powerful tool for investigating the size and structure of extragalactic radio sources (see Cohen, 1969 and references therein).

The ionosphere and interplanetary medium are nearby and the time-scale for variability is necessarily short, seconds or less. So the radio variability with 
a time-scale of years discovered in the first quasar, $3 \mathrm{C} 273$, at $\mathrm{cm}$ wavelengths (Dent 1965) was seen to be intrinsic to the source. Several decades of milliarcsecond resolution VLBI observations have confirmed the intrinsic nature of such variations and revealed a close relationship between structural changes on parsec-scales and flux density variations. The VLBI discovery of apparent super-luminal motion provided a natural explanation (Rees 1966) for brightness temperatures in excess of the $10^{12} \mathrm{~K}$ inverse Compton limit (Kellermann \& Pauliny-Toth 1969). However, systematic VLBI monitoring of internal proper motions of large numbers of compact radio sources has shown that the brightness temperatures will be limited to no more than $10-20$ times the $10^{12} \mathrm{~K}$ inverse Compton limit (Kellermann et al 2000).

In a series of careful flux density measurements at $3.2 \mathrm{GHz}$ Heeschen (1984) discovered short-term, small-amplitude flickering in a sample of flat-spectrum radio sources. More extensive multi-wavelength observations with the Bonn radio telescope detailed the presence of such short-term intra-day variability, IDV, in a number of flat-spectrum sources (Witzel et al 1987). While IDV has been observed in many sources at shorter wavelengths (see Wagner \& Witzel 1995 and references therein) it presents difficulties at $\mathrm{cm}$ and longer wavelengths because of the extreme brightness temperatures implied if the variations are intrinsic to the source.

\section{Evidence for Interstellar Scintillation}

Early IDV surveys revealed a Galactic latitude dependence which argued against an intrinsic origin and for refractive scintillation (Heeschen \& Rickett 1987). Further support for ISS came from the observed wavelength dependence of IDV. For the IDV source $0917+624$, the variations were found to be strongly correlated from 2.7 to $15 \mathrm{GHz}$, but with decreasing amplitude with increasing frequency; at $1.4 \mathrm{GHz}$ the IDV was smaller in amplitude and with a significantly longer characteristic time-scale (Rickett et al 1995). This phenomenon is seen in many other IDV sources, for example PKS 0405-385 (Kedziora-Chudczer et al 1997) and $\mathrm{J} 1819+3845$ (Dennett-Thorpe \& de Bruyn 2000) and is most easily explained as due to weak scattering at the higher frequencies and strong scattering at the lower frequencies (Walker 1998).

Direct evidence for ISS is provided by the observation of a significant timedelay between the arrival times of the pattern of variability in PKS 0405-385 seen at two widely spaced telescopes, the VLA in western USA, and the ATCA in eastern Australia (Jauncey et al 2000). Most recently, compelling evidence is found in the observations of a one-year signature in the long-term variability pattern in J1819+3845 (Dennett-Thorpe \& de Bruyn this Symposium). This annual cycle arises due to the variation of the ISM velocity as seen from the Earth in its cycle around the sun. Moreover, the same annual signature has been independently confirmed for 0917+624 (Jauncey \& Macquart A\&A submitted, Rickett et al ApJ submitted). The presence of Earth-related signatures, as seen here, makes it clear that refractive interstellar scintillation is the principal cause of IDV at these wavelengths in these sources. 


\section{Implied Source Structure and Brightness Temperature}

For ISS the angular scale depends on the scattering strength which is a function of frequency. In the weak scattering case, at a wavelength of $\lambda$ the angular size must be of the same order or smaller than the angular size of the first Fresnel zone, where $\theta=\sqrt{\lambda / 2 \pi D}$. The presence of ISS leads directly to implied microarcsecond source angular sizes and brightness temperatures well in excess of the $10^{12} \mathrm{~K}$ inverse Compton limit (eg Kedziora-Chudczer et al., 1997). These are considerably less extreme than the nanoarcsecond sizes and $10^{18}$ to $10^{21} \mathrm{~K}$ brightness temperatures were the variations intrinsic, but nonetheless remain uncomfortably high. Space VLBI $5 \mathrm{GHz}$ observations with VSOP (Preston et al., 2000, Lovell et al., 2000) already show directly the presence of unresolved cores with brightness temperatures well in excess of $10^{12} \mathrm{~K}$ in many flat-spectrum sources.

The distance, $\mathrm{D}$, to the equivalent scattering screen is a critical parameter in determining the brightness temperature of a scintillating source. For an unresolved source the brightness temperature scales as D (Walker 1998). For PKS 0405-385, a value of D 500 pc, half the Galactic scale height, leads to a $T_{b} \sim 5 \times 10^{14} K$ (Kedziora-Chudczer et al 1997). Using the velocity derived from the Earth's motion, Rickett et al (A\&A submitted) derive a screen distance of $170 \mathrm{pc}$ for $0917+614$, which implies a $T_{b}$ of $5 \times 10^{12} \mathrm{~K}$. Macquart et al (2000) derive a distance of $\sim 400 \mathrm{pc}$ in the direction of PKS $1519-273$ by making use of the observed scintillation time-scale and existing space VLBI observations.

Alternatively, for J1819+3845, Dennett-Thorpe \& de Bruyn (2000) find a distance of $20 \mathrm{pc}$, and argue that this is in agreement with the model of the local bubble (Bhat et al., 1998). They also suggest that the $T_{b}$ for PKS 0405-385 may be significantly reduced if $\mathrm{D}$ for this source were to be the same order as the $\sim 20$ pc derived for $1819+3845$. However, if $\mathrm{D}$ is set by the local bubble, then its geometry is such that for a source in the direction of PKS 0405-385, D would then be $150 \mathrm{pc}$. This still implies a brightness temperature in excess of $\sim 2 \times 10^{14} \mathrm{~K}$.

Reliable and accurate screen distances need to be determined for IDV sources before reliable brightness temperatures can be derived. Nevertheless, there remain strong arguments that brightness temperatures in excess of $10^{14} \mathrm{~K}$ are present, at least in some sources.

\section{Discussion}

Uncertainty remains as to the brightness temperatures encountered in IDV sources, with values possibly as high as $5 \times 10^{14} \mathrm{~K}$. Such high brightness temperatures require beaming factors that are well outside those expected from VLBI observations (Kellermann et al., 2000). Alternatively, if the scattering screen were closer than most estimates, the brightness temperatures may possibly be reduced to acceptable values.

However, the high brightness temperatures are not the only difficulties for synchrotron emission. The discovery of strong and variable circular polarization in the IDV source PKS 1519-273 (Macquart et al 2000) presents an additional 
problem for synchrotron theory, since they find no simple model that accounts for both the large amplitude and the observed frequency dependence.

In addition, several IDV sources, 0917+624 (Quirrenbach et al 2000) and PKS 1519-273 in particular, have been found to show IDV at $\mathrm{cm}$ wavelengths every time thay have been observed in detail over the past 10 - 15 years. These sources show only slow long-term variability, and the IDV characteristics have remained remarkably stable; the argument is not sustainable that the IDV is due to a sequence of separate outbutsts. Both sources exhibit brightness temperatures well in excess of the $10^{12} \mathrm{~K}$ inverse Compton limit, and it is difficult to see how such components do not cool in times much shorter than the observed lifetimes.

Acknowledgments. The Australia Telescope is funded by the Commonwealth Government for operation as a national facility by the CSIRO.

\section{References}

Bhat, R. N. D., Gupta, Y., \& Rao, P.A., 1998, ApJ, 500, 262

Dennett-Thorpe, J., \& de Bruyn, G., 2000, ApJ, 529, L65

Cohen, M.H., 1969, ARA\&A, 7, 619

Dent, W.A., 1965, Science, 148, 1458

Ginsburg, V.L., 1956, Soviet Phys. "Doklady", 1, 403

Heeschen, D.S., 1984, AJ, 89, 1111

Heeschen, D.S., \& Rickett, B.J., 1987, AJ, 93, 589

Jauncey, D.L., Kedziora-Chudczer, L., Lovell, J.E.J., Nicolson, G.D., Perley, R.A., Reynolds, J.E., Tzioumis, A.K., \& Wieringa, M.H., 2000, in Astrophysical Phenomena Revealed by Space VLBI, H. Hirabayashi, D.W. Murphy \& P.G. Edwards (eds), 147.

Kedziora-Chudczer, L., Jauncey, D.L., Wieringa, M.H., Walker, M.A., Nicolson, G.D., Reynolds, J.E. \& Tzioumis, A.K., 1997, ApJ, 490, L9

Kellermann, K.I., Vermeulen, R.C., Zensus, J.A., \& Cohen, M.H., 2000, in Astrophysical Phenomena Revealed by Space VLBI, H. Hirabayashi, D.W. Murphy \& P.G. Edwards (eds), 159

Lovell, J.E.J., et al., 2000, in Astrophysical Phenomena Revealed by Space VLBI, H. Hirabayashi, D.W. Murphy \& P.G. Edwards (eds), 183

Macquart, J-P., Kedziora-Chudczer, L., Rayner, D.P., \& Jauncey, D.L., 2000., ApJ, 538, 623

Preston, R.A., et al., 2000, in Astrophysical Phenomena Revealed by Space VLBI, H. Hirabayashi, D.W. Murphy \& P.G. Edwards (eds), 199

Quirrenbach, A., Kraus, A., Witzel, A., Zensus, J.A., Peng, B., Risse, M., Krichbaum, T.P., Wegner, R., \& Naundorf, C.E., 2000, A\&A Suppl., 141, 221

Rees, M.J., 1966, Nature, 211, 468

Rickett, B.J., Quirrenbach, A., Wegner, R., Krichbaum, T.P., \& Witzel, A., 1995, A\&A., 293, 479

Walker. M.A., 1998, MNRAS, 294, 307

Wagner, S.J., \& Witzel, A., ARA\&A, 33, 163

Witzel, A., et al., 1986, Mitt. Astron. Gesellschaft, 65, 239. 